\title{
Review
}

CARDIOLOGY

\section{Congenital Long and Short QT Syndromes}

\author{
Andrew J. Brenyo David T. Huang Mehmet K. Aktas \\ Department of Cardiovascular Diseases, University of Rochester Medical Center, Strong Memorial Hospital, \\ Rochester, N.Y., USA
}

\section{Key Words}

Long QT syndrome · Short QT syndrome •

Channelopathies · Arrhythmias

\begin{abstract}
Congenital long and short QT syndromes are familial arrhythmias characterized by derangement of repolarization and a high risk of sudden cardiac death due to ventricular tachyarrhythmias. With growing understanding of these syndromes in both the medical and lay communities, diagnostic and therapeutic difficulties are increasingly faced by health care providers. Modern genomics has determined the mechanism of arrhythmia induction in these patients, resulting in specific medical therapies and improved risk stratification. This paper reviews the common presentations, genetic etiology, basic evaluation, risk stratification, and therapeutic approach for both syndromes. Particular attention is paid to the effect of the individual syndrome on the cardiac action potential and its correlate the surface 12 lead ECG. In conclusion, patients with long and short QT syndromes are at risk for sudden death, with accurate diagnosis, risk stratification, and resulting appropriate therapy favorably altering their outcome.

Copyright $\odot 2012$ S. Karger AG, Basel
\end{abstract}

\section{Introduction}

Congenital long QT syndrome (LQTS) is a genetic condition characterized by a prolonged QT interval on the surface ECG and is associated with a high risk of sudden cardiac death (SCD) due to ventricular tachyarrhythmias. Mutations within 13 identified genes cause a variety of channelopathies affecting myocardial repolarization, resulting in QT prolongation [1]. Conversely, gain-of-function mutations in a specific few of these genes result in shortening of the QT interval and myocardial repolarization. Short QT syndrome (SQTS) is characterized by constantly short QTc intervals $(<300 \mathrm{~ms})$ and a high familial incidence of palpitations, syncope, SCD, and atrial fibrillation [2]. In this review article we present common clinical scenarios of patients with LQTS and SQTS, review their pathophysiology, and discuss the diagnostic workup and subsequent treatment options. Future and emerging therapeutic options for patients with congenital LQTS and SQTS are also mentioned.

\section{Common Clinical Presentations}

Patients with LQTS and SQTS will present along a spectrum of illness ranging from an incidental ECG finding in an asymptomatic patient to an abnormal QT inter-

\section{KARGER \\ Fax +4161306 1234 \\ E-Mail karger@karger.ch}

www.karger.com
(C) 2012 S. Karger AG, Basel

$0008-6312 / 12 / 1224-0237 \$ 38.00 / 0$

Accessible online at:

www.karger.com/crd
Andrew J. Brenyo, MD

University of Rochester Medical Center, Strong Memorial Hospital

Department of Cardiovascular Diseases, 601 Elmwood Avenue, Box 679-C

Rochester, NY 14642 (USA)

Tel. +1 585275 4776, E-Mail Andrew_Brenyo@urmc.rochester.edu 
val post-cardiac arrest. Any history of arrhythmic syncope (drop attacks), family history of SCD, or inherited arrhythmias should be sought. Beyond the most commonly encountered incidentally identified QT abnormalities, discovery of abnormal QT intervals upon ECG screening after the onset of syncope or the diagnosis of a family member are likely the most frequent reasons for presentation to a cardiovascular specialist.

Historically, atypical initial presentations of patients eventually diagnosed with LQTS, such as a long-standing history of syncope and recurrent 'seizures' despite antiepileptic therapy, have not been uncommon. Classic situational arrhythmic/syncopal triggers have been well described to be associated with specific LQTS types and should be investigated upon initial presentation. Arrhythmic events associated with LQT1 are triggered by swimming or other exercise, whereas most events in LQT2 and LQT3 are associated with acute arousal or sleep/rest without arousal [3-5].

SQTS presentation differs from LQTS in that the symptomatic patient will often note onset of atrial fibrillation and palpitations at a young age potentially independent of a history of syncope [6]. Also in contrast to LQTS, no specific situational arrhythmic triggers have been identified for the SQTS phenotype and the terminal ventricular arrhythmia is typically ventricular fibrillation in SQTS and torsades de pointes in LQTS.

\section{Epidemiology}

Historically, congenital LQTS was thought to be a rare disorder in part because only patients with the most severe QT prolongation were detected and reported. However, it is estimated that at least $1 / 2,500$ to $1 / 7,000$ people worldwide are affected with LQTS [7]. As awareness and screening for LQTS improves, its prevalence is expected to increase. Insufficient data is available to suggest worldwide, racial, or ethnic variation in prevalence. LQTS is more commonly diagnosed in women, potentially inaccurately secondary to the sex-based differences in the upper limit for the QTc interval in post-pubertal females compared to males (460 and $440 \mathrm{~ms}$, respectively) [8].

Congenital short QT syndrome was first reported as a cause for increased risk of sudden death in 2000 [2], and at this time determinations of its incidence and prevalence are difficult to make due to limited data. With the increased use of patient registries such as the European SQTS Registry [9], characterization of the prevalence and incidence of SQTS should be possible.

\section{Etiology}

\section{Long QT Syndrome}

Congenital LQTS is inherited as a monogenic disorder with primarily autosomal dominant inheritance and variable penetrance [10]. About $85 \%$ of the reported cases are inherited from one of the parents, with the remaining $15 \%$ of affected patients having de novo mutations. Mutations identified in 13 different genes account for congenital LQTS, with LQT1, LQT2, and LQT3 mutations constituting $90-95 \%$ of cases (table 1) [1]. These mutations cause alterations in specific ion channels active during the normal action potential (fig. 1a) leading to the pathophysiologic alteration of repolarization, manifested as a prolongation of the QT interval on the ECG.

LQT1 results from a loss-of-function mutation in the KCNQ1 gene which encodes the alpha-subunit of the slow activating potassium channel responsible for the IKs current. KCNQ1 mutations are the most commonly identified in genotyped patients. A homozygous mutation in KCNQ1 results in the rare autosomal recessive Jervell and Lange-Nielsen syndrome (JLNS) $[11,12]$ that is associated with congenital deafness and a high rate of fatal cardiac events [13]. Dysfunctional IKs channels lead to dispersion of repolarization (fig. 1b) from the epicardial to the endocardial surface, potentiating the development of ventricular tachyarrhythmias. Electrocardiographically, there is a characteristic prolonged QT interval associated with a broad based T wave (fig. 2).

The LQT2 phenotype arises from mutations in the $\mathrm{KCNH} 2$ gene encoding the alpha (HERG) subunit of the potassium channel responsible for the $\mathrm{IKr}$ current. $\mathrm{KCNH} 2$ mutations are the second most commonly identified, accounting for up to 35-40\% of genotyped patients $[7,14]$. Dysfunctional IKr channels (fig. 1b) lead to slowing and transmural dispersion of repolarization which predisposes patients to ventricular tachyarrhythmias, particularly torsades de pointes. Electrocardiographically, a prolonged QT interval is seen with characteristic low amplitude and notched $\mathrm{T}$ waves often visualized best in the limb leads or rhythm strip (fig. 3).

LQT3 stems from gain-of-function mutations in the SCN5A gene which encode for rapidly inactivating sodium channels, allowing an inward flow of sodium ions to persist long into the plateau phase of the action potential and thereby prolonging repolarization (fig. 1b). Electrocardiographically, there are characteristic long ST segments with a late appearing $\mathrm{T}$ wave resulting in a long QT interval (fig. 4). 

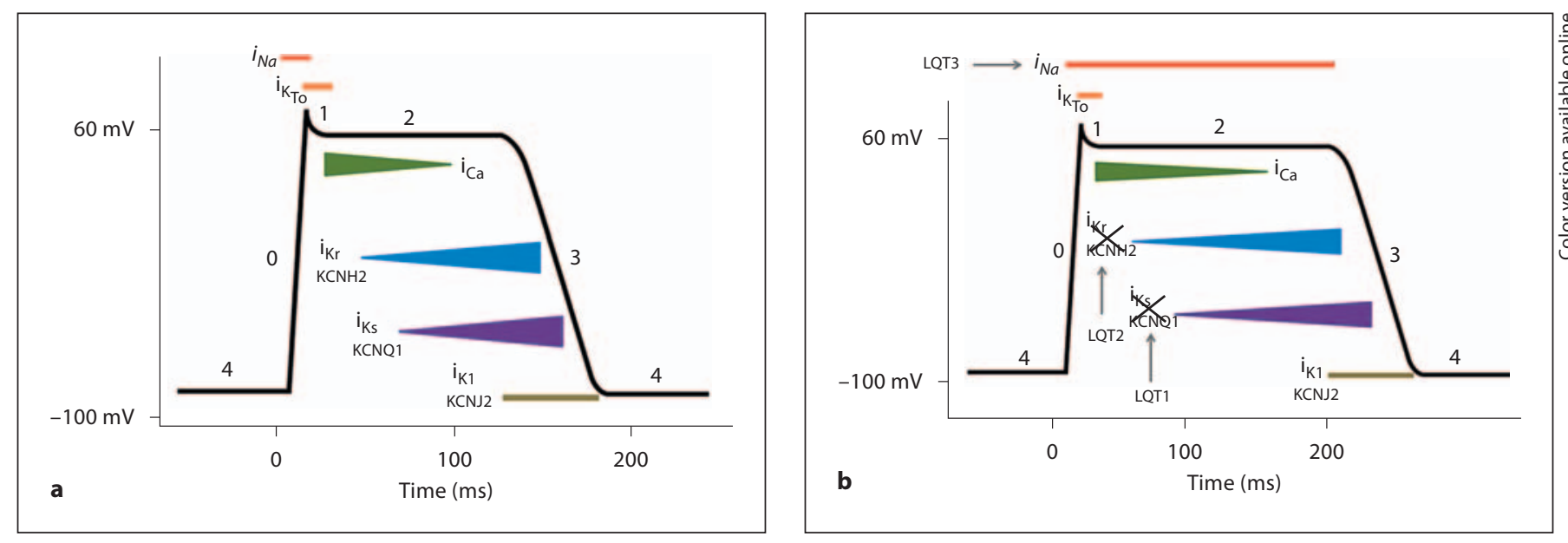

Fig. 1. a Normal cardiac action potential. Inward currents are presented in italics and outward currents are in normal font. b Individual LQTS mutations and their effect on the cardiac action potential. Individual currents are represented as inward (italicized font) and outward (normal font). LQTS 1, 2, and 3 are presented with the individual repolarizing current effected for each syndrome indicated. Of note, the action potential is markedly prolonged secondary to the loss (or gain) of the individual currents that otherwise would act to end it. c Individual SQTS mutations and their effect on the cardiac action potential. Individual currents are represented as inward (red/solid arrows) and outward (blue/dashed arrows). Increased or new currents are presented with bold arrows. SQT 1, 2, and 3 syndromes are presented with the individual repolarizing current effected for each syndrome indicated. Of note, the action potential is markedly shortened secondary to the gain of the individual currents.

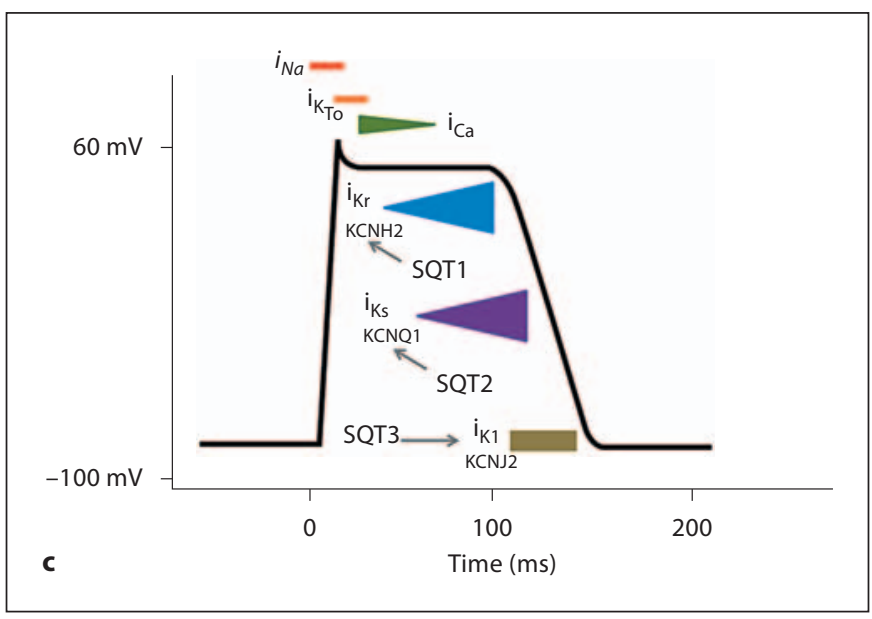

Table 1. Currently identified LQTS gene mutations

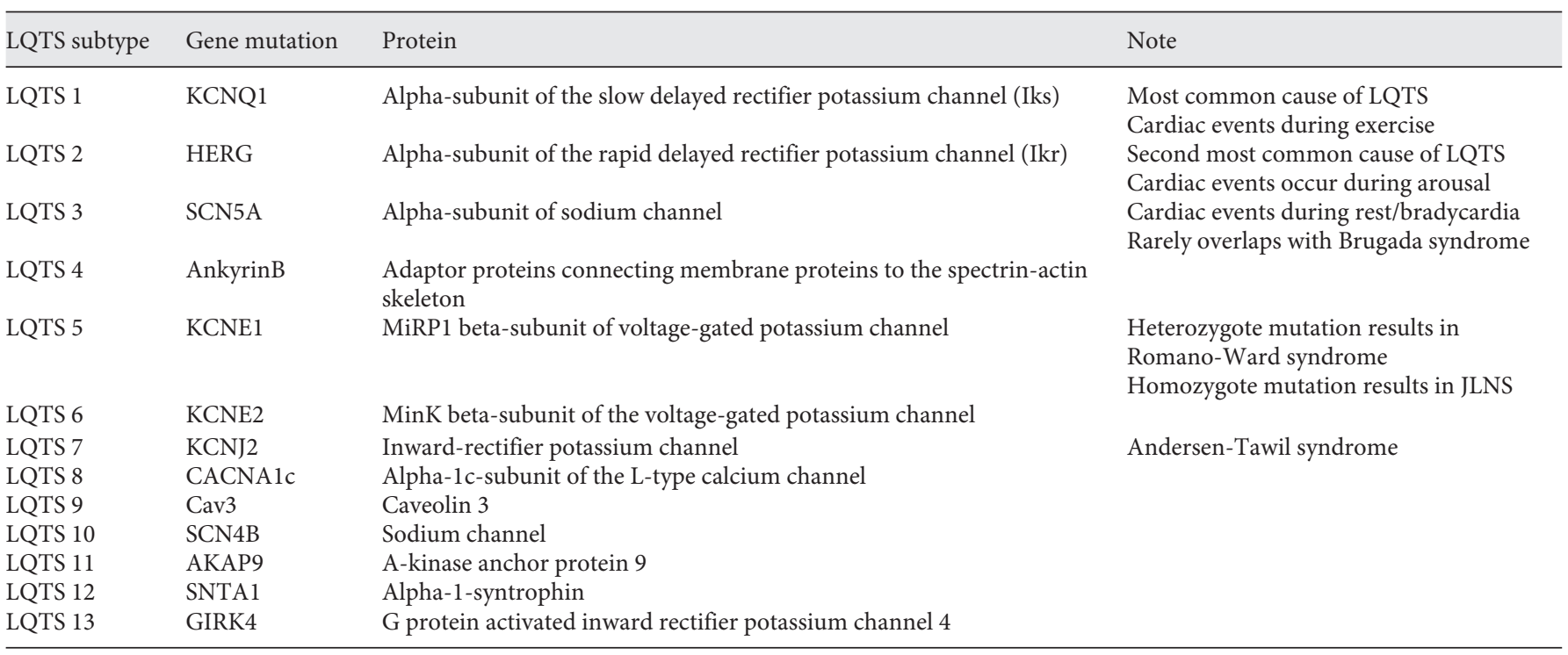




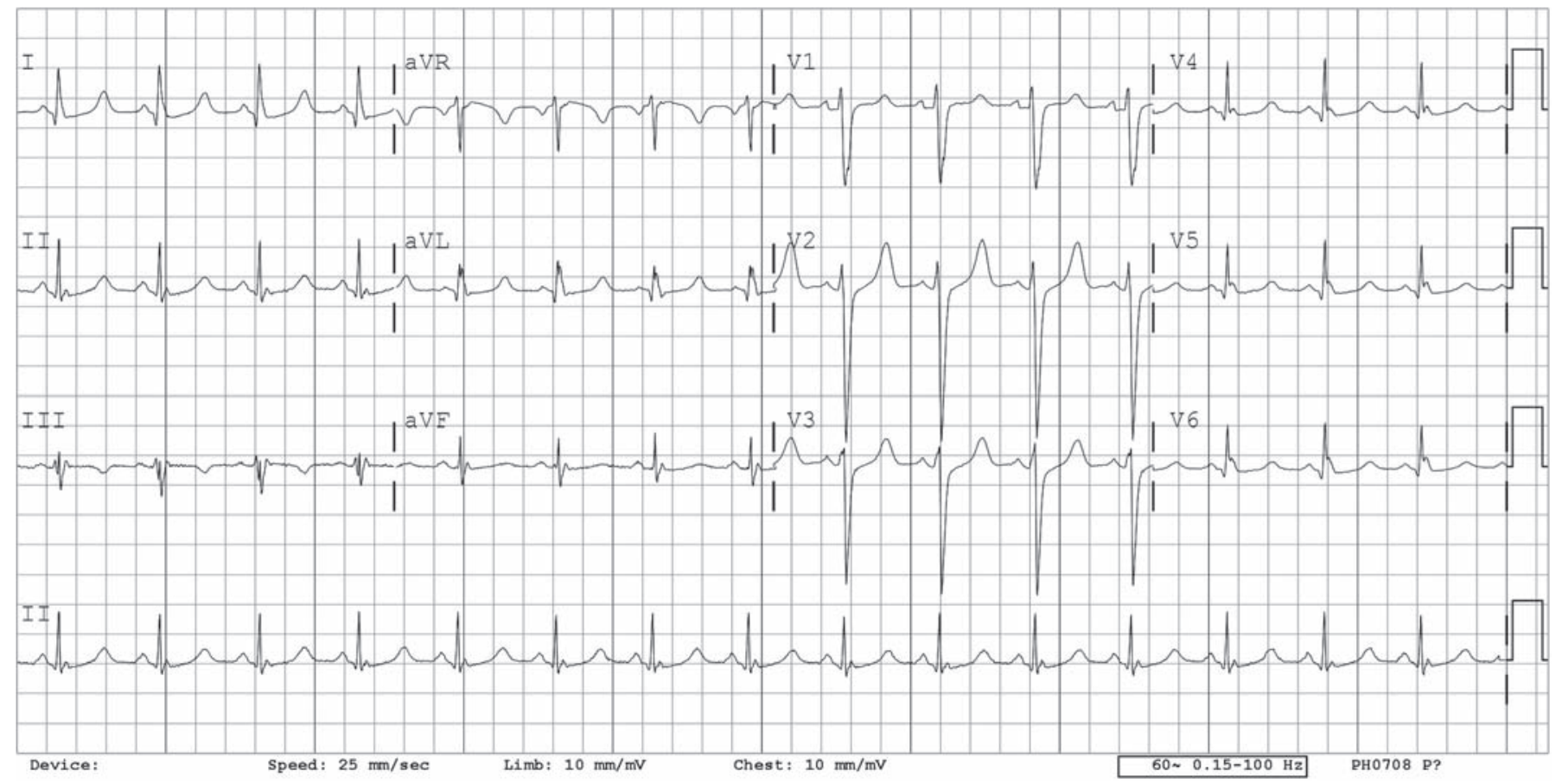

Fig. 2. Representative ECG of a patient with congenital LQTS type 1. Note broad based, peak T waves producing QT prolongation.

Fig. 3. Representative ECG of a patient with congenital LQTS type 2 . Note the notched T waves particularly in lead II and the rhythm strip at the bottom of the ECG.

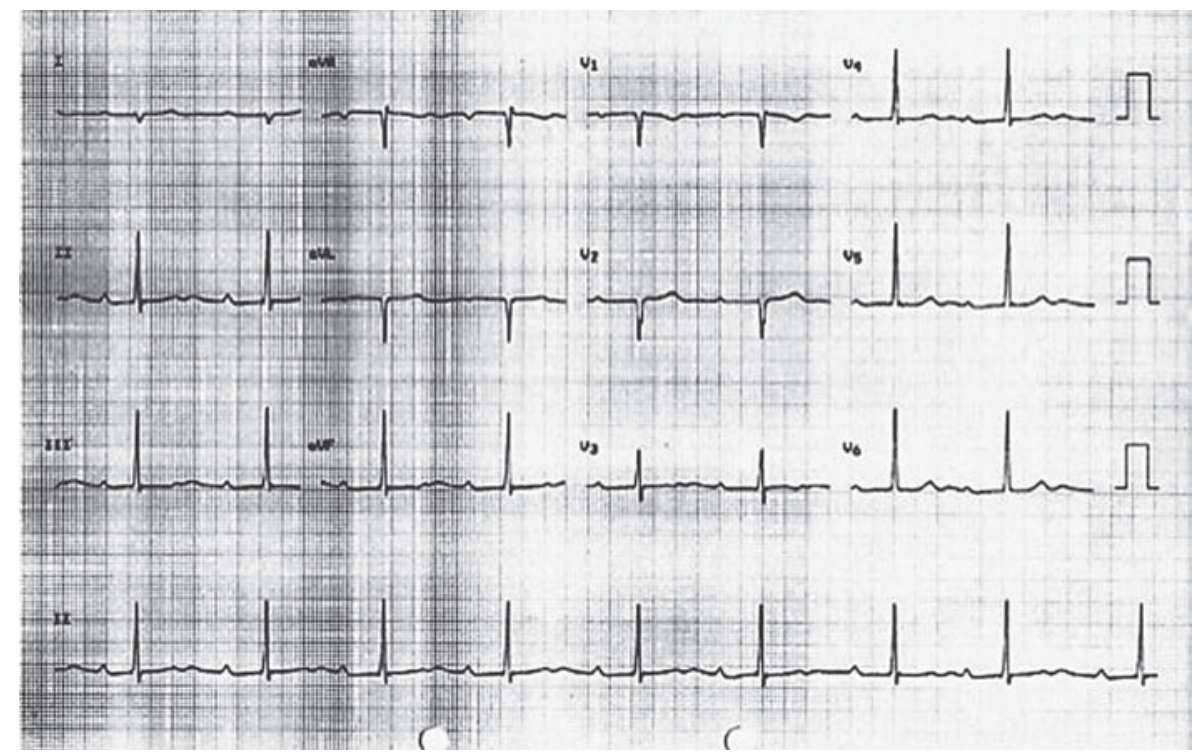

LQT4-LQT13 have been described but are responsible for less than $10 \%$ of all genotyped cases and are typically encountered only in individual families (table 1) [7].

LQTS can be phenotypically classified into 3 congenital syndromes. Romano-Ward syndrome is inherited as an autosomal dominant trait and is the most common form of LQTS. It may result from a mutation in any one of 13 identified genes and is not associated with deafness; rather, it represents the common LQTS phenotypes. JLNS is inherited as an autosomal recessive trait and results from a 


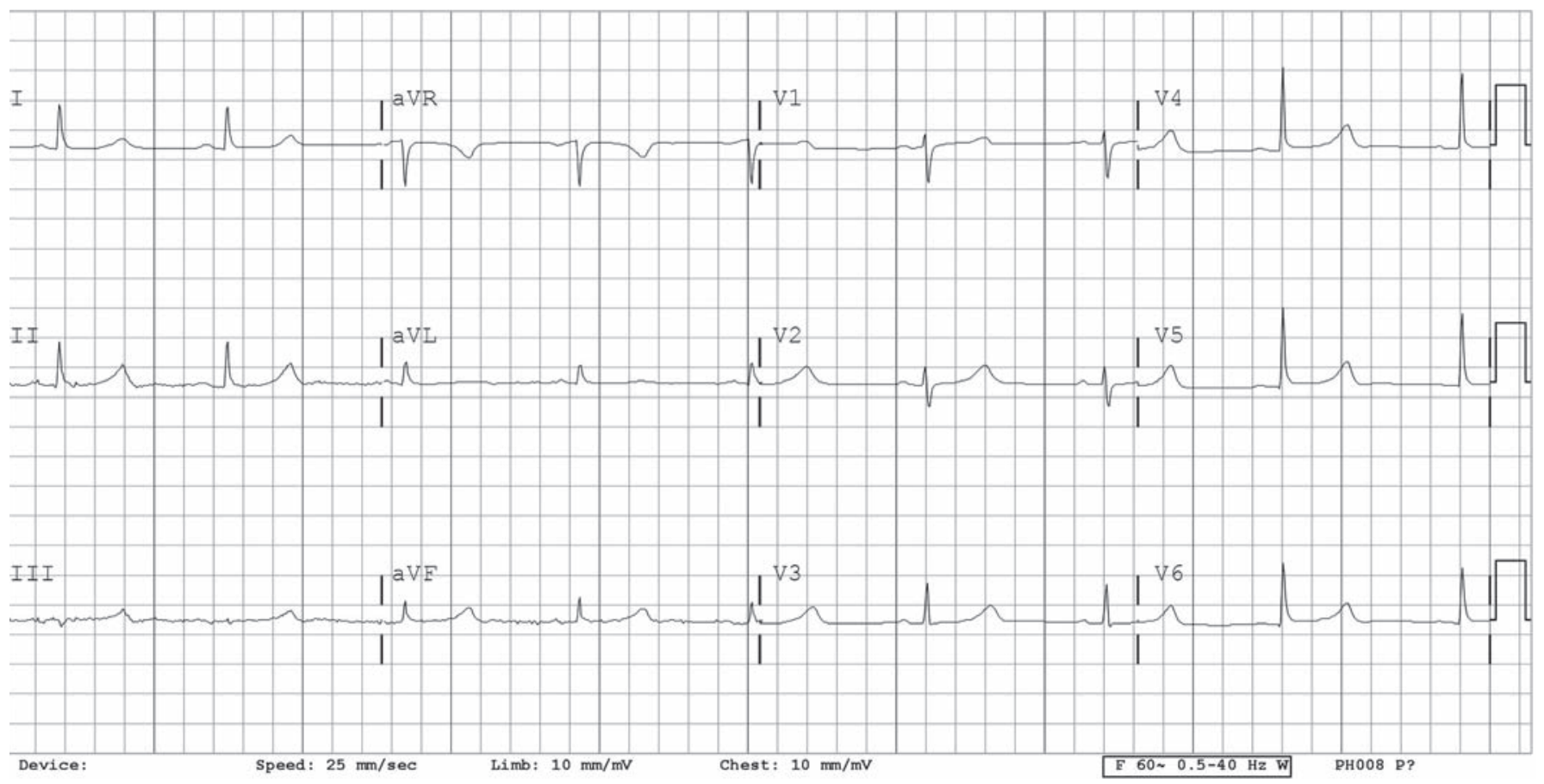

Fig. 4. Representative ECG of a patient with congenital LQTS type 3. Note the late appearance of the T wave, after an isoelectric, long ST segment.

homozygous mutation in KCNQ1 [11]. It is clinically characterized by a very severe form of LQTS and is associated with sensorineural deafness. Andersen-Tawil syndrome, also known as hypokalemic periodic paralysis or LQT7, is a rare autosomal dominant condition resulting from a mutation in KCNJ2 $[15,16]$. These patients suffer from periodic paralysis and ventricular tachyarrhythmias and have a variety of dysmorphic features, including micrognathia, low set ears, widely spaced eyes, and clinodactyly.

\section{Short QT Syndrome}

Congenital SQTS results from autosomal dominant gain-of-function mutations in potassium channels during phases 2 and 3 of the action potential (fig. 1c) resulting in rapid cardiac repolarization. Currently we know significantly less about the genetics of SQTS compared to LQTS secondary to the rarity of SQTS. The syndrome is characterized in the few patients identified so far by a shortened QT interval of less than 300-325 ms after correction for heart rate at rates below 80 beats/min (fig. 5). A number of gain-of-function mutations have been identified within the three culprit voltage-gated potassium channel genes: KCNH2, KCNQ1, and KCNJ2. The gain of function results in a greater than normal efflux of potassium from the cell during the repolarization phase and a shortening of the action potential. SQTS has been separated into three types based upon the culprit mutation (when known): (1) the most common, KCNH2, labeled short QT1; (2) KCNQ1, short QT2, and (3) KCNJ2, short QT3. KCNH2 encodes IKr, which is responsible for the rapid component of the delayed rectifier potassium current. The remaining two genes implicated in short QT syndrome (KCNQ1 and KCNJ2) have, to date, been identified only in single index cases $[17,18]$. Probands reported in the literature are genotype positive for one of the three described mutations (KCNQ1, KCNJ2, and KCNH2) 21\% of the time, indicating that a significant proportion of causative genetic mutations remain to be identified [19].

\section{Diagnostic Approach}

The diagnostic process is similar for both LQTS and SQTS, resting primarily on historic, electrocardiographic, and genetic data with a limited role for exercise and invasive electrophysiologic testing.

\section{Long QT Syndrome}

A careful focused history is necessary for the diagnosis of LQTS and potentially may even identify its subtype. 


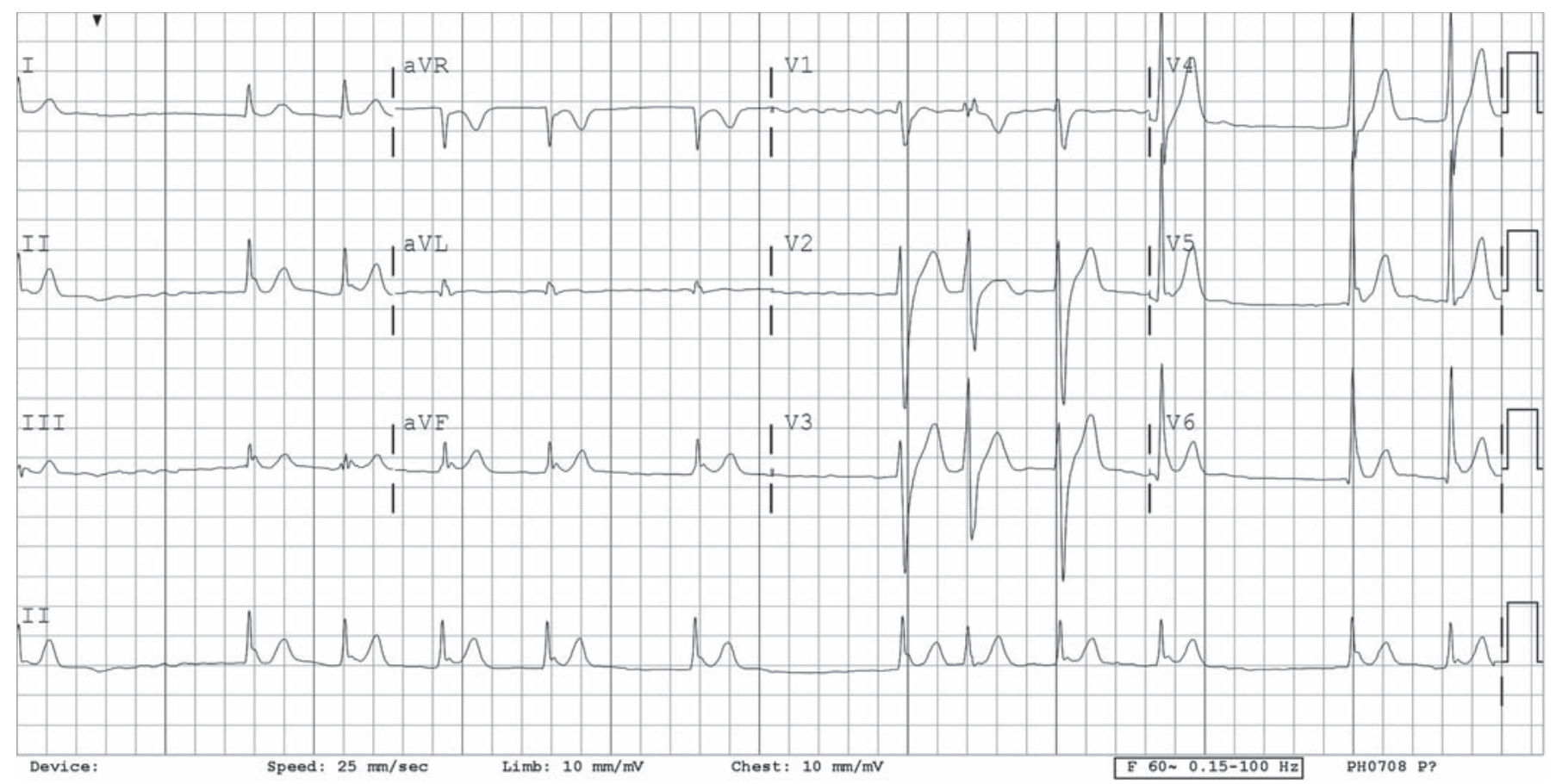

Fig. 5. Representative ECG of a patient with congenital SQTS. Note the presence of atrial fibrillation and the absence of an ST segment.

Particular attention should be paid to any episode triggers such as swimming, exercise, or arousal from sleep, all of which have classically been associated with individual LQT syndromes as discussed previously. A thorough family history with focus on any episodes of drowning, SCD, or inherited arrhythmia may be important to understand the penetrance and severity of the condition. Furthermore, the history may help elucidate potential benign causes for syncope, some of which may be completely reversible.

The electrocardiographic diagnosis of LQTS is not typically straightforward as nearly $2.5 \%$ of the normal population may have a mildly prolonged QT interval, and approximately $25 \%$ of genotype-positive LQTS patients have a normal QT interval [20]. However, the resting ECG remains crucial in the diagnosis of LQTS and should be undertaken in all suspected cases. Additionally, in a patient with documented LQTS, it is very important to obtain ECGs from parents, siblings, and any family members with pre-syncope or syncope. Developing a family pedigree utilizing this information may aid in the discovery of affected but currently asymptomatic undiagnosed family members.

Exercise treadmill testing may help to identify abnormal QT interval prolongation during exercise and into recovery. Such derangements are helpful in diagnosing LQTS, particularly LQT1 in which the QT interval and QTc increase with exercise (typically an event trigger in this population) more than those of controls or other LQT types [21]. Sy et al. [22] recently utilized an exercisebased algorithm for the diagnosis of LQT in asymptomatic relatives of LQTS probands. The combination of resting and 4-min recovery QTc in their screening algorithm yielded a sensitivity of 0.94 and a specificity of 0.90 for detecting LQTS carriers, with similar results noted in the validation cohort [22]. Furthermore, for suspected LQTS carriers (based upon a resting QTc of $>470 \mathrm{~ms}$ ) the 1-min recovery QTc allowed the authors to determine if the carrier was LQT1 $(\geq 460 \mathrm{~ms})$ or LQT2 $(<460 \mathrm{~ms})$ genotype positive [22]. Exercise testing may also assist in the prescription of a maximum exercise level for patients presenting with exercise-induced symptoms of presyncope or syncope by simulating similar circumstances in a controlled environment.

Particularly in the setting of a concerning phenotype but normal resting and exercise QTc intervals, administration of the natural sympathetic agonist epinephrine has been utilized to unmask diagnostic changes in ventricular repolarization for LQT1 and LQT2 patients [2326]. Three epinephrine administration techniques, i.e. 
the Mayo [24], Shimizu [25], and Hekkala [26] protocols, have been reported in the literature, with similar efficacy [26]. The change in QTc, QT apex, and T wave peak durations from baseline and during epinephrine administration are calculated. The pattern of change within these parameters during provocation including shortening of the QT apex and lengthening of T wave peak and QTc intervals identifies genotype-positive patients. The Shimizu protocol has also utilized change in QTc with peak epinephrine infusion to correctly identify LQT2 genotype-positive patients $[23,25]$. As of yet, epinephrine testing has not proven useful in identifying LQT3 patients and when the index of suspicion for the LQT3 genotype is high, sympathetic provocation should be forgone in favor of genetic testing [23].

Genetic testing may allow the determination of a patient's LQTS subtype through the identification of causative mutations (table 1). Knowledge of the specific gene mutation can be used in risk stratification of patients with congenital LQTS and in the prescription of medical therapy [14, 27]. Recent Heart Rhythm Society, European Heart Rhythm Association, and Canadian Heart Rhythm Society consensus statements support the use of genetic testing for symptomatic patients (post-SCD or syncope) with abnormal QTc intervals, asymptomatic patients with consistent idiopathic prolongation of the QTc interval or during provocative testing and asymptomatic firstdegree relatives of genotype-positive LQT probands [28, 29]. In its current state, genetic testing is an imperfect tool due to its focus on the most common known mutations resulting in low sensitivity and its high cost. However, it remains a cornerstone of the diagnostic evaluation and risk stratification of potential LQTS patients. In contrast, investigating for inducible ventricular arrhythmias using electrophysiology studies has not been shown to have significant value in the diagnosis of patients with suspected LQTS [30].

\section{Short QT Syndrome}

Similar to LQTS, the evaluation of SQTS begins with a comprehensive history of events (syncope, presence of atrial fibrillation, and SCD) both in the patient and within the family. ECG evidence of the phenotype (short QT interval plus or minus atrial fibrillation) is also necessary for diagnosis. In contrast to LQTS, the role of genetic testing is limited in SQTS as most patients who are phenotype positive currently have unknown genetic mutations. Patients who present with electrocardiographic evidence of the short QT phenotype along with symptoms (AF or SCD) should be tested for the three most common muta- tions (KCNH2, KCNQ1, and KCNJ2). The reason for this testing, even in the face of the majority of causative mutations being unknown, is primarily to determine the type of SQTS for both prognostic and therapeutic purposes.

Patients with congenital SQTS have very short atrial and ventricular refractory periods and ventricular fibrillation can be easily induced with programmed ventricular stimulation [31]. Consistent between LQTS and SQTS, an electrophysiology study to assess inducibility of ventricular tachycardia or ventricular fibrillation by programmed electrical stimulation is not necessary to establish a diagnosis.

\section{Risk Stratification}

\section{Long QT Syndrome}

Risk stratification of patients with LQTS plays a central role in guiding therapy after diagnosis. Accumulating data from the International LQTS Registry has facilitated a comprehensive analysis of risk factors for aborted cardiac arrest or SCD in multiple prespecified age groups $[20,32-35]$. These analyses have consistently indicated that the phenotypic expression of LQTS is time dependent and age specific, warranting continuous risk assessment in affected patients.

Utilizing the data from the International LQTS Registry studies, patients with LQTS are risk stratified for the probability of a future cardiac event (syncope or SCD) primarily according to LQT genotype $[13,32,34]$ (when identified), mutation location [35] (when identified), and QTc interval length $[20,32,36]$, followed by sex [33, 34, 37], age $[33,34,37]$, and number and type of events. A family history of SCD in a first-degree relative of genotype-positive LQT patients has not been shown to be a significant predictor of adverse outcomes in populations that are of mixed LQT genotype but predominately LQT1 [33].

Low risk patients (probability of first cardiac event $<30 \%$ ) include men or women with LQT1 and QTc $<500$ $\mathrm{ms}$ and men with LQT2 and QTc $<500 \mathrm{~ms}$. Intermediate risk patients (probability of first cardiac event 30-49\%) include women with LQT2 and QTc $<500 \mathrm{~ms}$, men with LQT3 and QTc $<500 \mathrm{~ms}$, and women with LQT3. High risk patients (probability of first cardiac event $\geq 50 \%$ ) include men or women with LQT1 or LQT2 and QTc $\geq 500$ $\mathrm{ms}$ and men with LQT3 and QTc $\geq 500 \mathrm{~ms}$ [14]. Adequate risk stratification, particularly in young asymptomatic genotype-positive patients, is very complex secondary to the morbidity of implantable cardioverter-defibrillator (ICD) therapy weighed against the risk of SCD. 
Asymptomatic LQTS patients have a lower event rate per year or per decade than patients with a prior cardiac event $[7,33,37,38]$. Patients with prior syncope have a much higher likelihood of future events including SCD $[37,38]$. In early childhood, boys with LQT1 are more likely to suffer syncope or sudden death, but less likely than girls to have symptoms later in life [39]. This gender risk reversal has been recognized and reported in all patients with LQTS, where male patients with LQTS are at higher risk for events up to the age of 15 [37], after which women appear to be at greater risk $[34,39,40]$. Women with LQT2 appear to be at higher risk for cardiac arrest, syncope, and/ or sudden death than males and remain at risk into adulthood $[14,41]$, while males with LQT3 are more likely to have events than are their female counterparts [14]. The increased risk of cardiac events in post-pubertal women ongoing through menopause supports a currently poorly understood hormonal contribution to abnormalities in ventricular repolarization and arrhythmogenesis in this population [32]. Overall, the number of deaths in patients with LQTS is greater in women than in men [42].

\section{Short QT Syndrome}

As most patients who are phenotype positive have currently unknown genetic mutations, the role of genetic testing is limited. Patients who present with the short QT phenotype (short QT on ECG and symptoms) should be tested for the three most common mutations noted above. Patients who test positive for KCNJ2 are at low risk for future adverse events and can forgo further therapy, whereas those who test positive for KCNQ1 are more likely to experience arrhythmic events and should be considered for more aggressive, device-based therapy [43]. Given that congenital short QT syndrome is a relatively newly recognized clinical syndrome, beyond such genespecific risk stratification, little in the way of data is available regarding clinical risk stratification. As a rough rule of thumb, however, patients with SQTS who have suffered syncope are at high risk for recurrent events and should be considered for aggressive medical and device therapy.

\section{Treatment Approach}

The treatment scheme is similar for both of these congenital arrhythmias, despite their different pathophysiology and genetic origin. High risk LQTS patients, those post-cardiac arrest or with numerous arrhythmic syncopal episodes, and all SQTS patients should be treated aggressively with ICD placement. This is typically in addi- tion to available medical therapy (beta-blockers for LQTS and quinidine for SQTS) to prevent future episodes. Intermediate or low risk LQTS patients are often managed medically and observed longitudinally for persistent symptoms.

\section{Long QT Syndrome}

Given the increasing prevalence of congenital LQTS and the associated risk of SCD, health care providers are likely to find themselves encountering challenging management decisions. The mainstay of treatment for all patients with LQTS is lifestyle modification and beta-blocker therapy [37, 44-46] with the implantation of an ICD when medical therapy fails or with a presenting cardiac arrest [47]. These three more common and other less frequently utilized therapies for LQTS are discussed below.

\section{Lifestyle Modification}

Competitive sports or extreme exertion should be avoided by all patients with congenital LQTS [48]. Possible exceptions may include golf, curling, cricket, billiards, or bowling [48]. Even noncompetitive swimming, especially for LQT1 patients, must be limited and if performed should be done so under close supervision. Patients with LQT2 should avoid startling acoustic stimulation such as alarm clocks [3]. Electrolyte losses due to vomiting, diarrhea, or excessive sweating should be replaced in order to avoid hypokalemia and hypomagnesemia that may exacerbate prolongation of the QT interval. In addition, LQTS patients must avoid medications known to prolong the QT interval with particular attention paid to antibiotic, antiemetic, antiepileptic, antiarrhythmic, antipsychotic, and antidepressant medication classes (see www.qtdrugs.org).

\section{Beta-Blockers}

The mainstay of medical therapy for all patients with LQTS involves beta-blocker therapy [49]. As ventricular arrhythmias may arise during a state of high adrenergic tone and increase the occurrence of after-depolarizations, beta-blockers are used to blunt adrenergic stimulation. Beta-blockers themselves do not shorten the QT interval; rather, they act to minimize the risk of triggered activity through modulation of the cardiovascular adrenergic tone. Unfortunately, beta-blockers provide less protection to patients with LQT3, as has been described pre- 
viously [50]. Even though no randomized controlled trials exist, observational data suggest a strong mortality benefit with beta-blocker therapy [49]. The efficacy of beta-blockade may be assessed with exercise tolerance testing to ensure that there is blunting of the heart rate response during exertion.

\section{Implantable Cardioverter-Defibrillator}

Use of an ICD in conjunction with beta-blockers has proven invaluable in the treatment of patients with LQTS suffering ongoing symptoms despite beta-blocker therapy $[47,51,52]$. ICDs are now considered first line therapy for patients who have had a previous cardiac arrest, those with recurrent arrhythmic syncope despite beta-blocker therapy $[52,53]$, patients who cannot tolerate beta-blockers or in whom beta-blockers are contraindicated, and high risk patients (QTc $>500 \mathrm{~ms}$ in men/women with LQT1 and LQT2 and in men with LQT3) and patients with a QTc $>550 \mathrm{~ms}$.

\section{Sympathectomy}

Left cervicothoracic sympathectomy involves surgical resection of the lower half of the left stellate ganglion along with several other thoracic ganglia in an attempt to denervate the heart [54]. This procedure, which can now be performed in a minimally invasive manner [34], remains an option for patients intolerant of beta-blockers, those with recurrent cardiac events on beta-blockers, patients receiving multiple ICD shocks, and patients in whom an ICD implant is not feasible due to either physical or psychosocial limitations.

\section{Permanent Pacemaker Implant}

When combined with beta-blockers, ventricular pacing, which prevents bradycardia, may facilitate the titration of beta-blockers to more effective antiarrhythmic doses and can also serve to prevent pause-dependent torsades de pointes $[55,56]$. However, no randomized study exists comparing the efficacy of pacemakers combined with beta-blockers to ICDs in preventing symptoms in patients with LQTS and this approach is only utilized in conjunction with ICD implantation.

The Long and Short of QT Syndromes

\section{Emerging Therapy}

Ranolazine is an antianginal agent that is approved for the treatment of chronic ischemic chest pain [57]. Ranolazine has several effects on ion channels, but its primary effect is reduction of the late sodium current (late Ina), a depolarizing current which contributes to action potential prolongation [57]. Preliminary studies have shown significant QT interval shortening when ranolazine is given as an infusion to patients with LQT3 [58]. Ranolazine is not currently approved for the treatment of LQTS and studies of ranolazine in patients with LQTS are ongoing.

Flecainide is a class IC antiarrhythmic drug with potent sodium channel-blocking properties. Patients with LQT3 mutations have an abnormal sodium channel allowing an inward current to persist long into the plateau phase of the action potential, thereby prolonging the QT interval. In these patients, flecainide has been shown to shorten and even normalize the QT interval [59]. However, flecainide may increase the risk of SCD in patients with overlapping LQT3 and Brugada syndrome, making its routine use not recommended [60]. Furthermore, there is no evidence that flecainide or other sodium channel blockers reduce mortality in LQT3.

\section{Short QT Syndrome}

Patients with congenital SQTS have a very high risk of sudden death and for this reason ICD implantation has been recommended as first line therapy $[6,31,43]$. However, it should be stressed that secondary to our limited experience with SQTS the therapeutic options are limited beyond ICD implants. Of course, device implantation in young patients presents numerous technical as well as psychosocial issues that may make device implantation prohibitive. Genetic testing, when positive for known SQTS mutations, can provide further guidance regarding this often difficult decision. Asymptomatic patients with SQTS who have a positive KCNJ2 mutation are less likely to have adverse events and may forego ICD implants, whereas those who test positive for $\mathrm{KCNH} 2$ are more likely to experience arrhythmic events and should receive more aggressive, device-based therapy $[18,19,61]$. As the role of genetic information is usually limited (as few patients are genotype positive for the described mutations) the decision to pursue ICD implantation often remains difficult for clinicians and families.

$\mathrm{KCNH} 2$-positive patients may also be considered for antiarrhythmic therapy with quinidine, as originally described by Gaita et al. [43]; it normalized the QT interval 
and rendered VT/VF noninducible at EP study after ICD implantation. In contrast, patients who were not KCNH2 positive did not experience normalization of the QT interval or loss of ventricular arrhythmia inducibility.

\section{Conclusion}

Patients with congenital LQTS and SQTS are at risk for sudden death. Modern genomics has shed light on the mechanism of arrhythmia induction in these patients and has allowed for their further risk stratification. As the number of described mutations increases for both syndromes (particularly for SQTS) it may be possible to identify responsible mutations in all phenotype-positive patients, improving risk stratification and simplifying therapeutic decision making. Accurate diagnosis and institution of effective contemporary medical and/or device therapy can favorably improve outcomes in these patients.

\section{Conflict of Interest}

The authors have nothing to disclose.

\section{References}

1 Hedley PL, Jorgensen P, Schlamowitz S, et al: The genetic basis of long QT and short QT syndromes: a mutation update. Hum Mutat 2009;30:1486-1511.

2 Gussak I, Brugada P, Brugada J, et al: Idiopathic short QT interval: a new clinical syndrome? Cardiology 2000;94:99-102.

- 3 Schwartz PJ, Priori SG, Spazzolini C, et al: Genotype-phenotype correlation in the long-QT syndrome: gene-specific triggers for life-threatening arrhythmias. Circulation 2001;103:89-95.

-4 Moss AJ, Robinson JL, Gessman L, et al: Comparison of clinical and genetic variables of cardiac events associated with loud noise versus swimming among subjects with the long QT syndrome. Am J Cardiol 1999;84: 876-879.

5 Ackerman MJ, Tester DJ, Porter CJ: Swimming, a gene-specific arrhythmogenic trigger for inherited long QT syndrome. Mayo Clin Proc 1999;74:1088-1094.

6 Gaita F, Giustetto C, Bianchi F, et al: Short QT syndrome: a familial cause of sudden death. Circulation 2003;108:965-970.

7 Goldenberg I, Zareba W, Moss AJ: Long QT syndrome. Curr Probl Cardiol 2008;33:629_ 694.

8 Imboden M, Swan H, Denjoy I, et al: Female predominance and transmission distortion in the long-QT syndrome. N Engl J Med 2006;355:2744-2751.

-9 Giustetto C, Schimpf R, Mazzanti A, et al: Long-term follow-up of patients with short QT syndrome. J Am Coll Cardiol 2011;58: 587-595.

10 Priori SG, Napolitano C, Schwartz PJ: Low penetrance in the long-QT syndrome: clinical impact. Circulation 1999;99:529-533.

11 Jervell A, Lange-Nielsen F: Congenital deafmutism, functional heart disease with prolongation of the Q-T interval and sudden death. Am Heart J 1957;54:59-68.
12 Schwartz PJ, Spazzolini C, Crotti L, et al: The Jervell and Lange-Nielsen syndrome: natural history, molecular basis, and clinical outcome. Circulation 2006;113:783-790.

13 Goldenberg I, Moss AJ, Zareba W, et al: Clinical course and risk stratification of patients affected with the Jervell and Lange-Nielsen syndrome. J Cardiovasc Electrophysiol 2006; 17:1161-1168.

14 Priori SG, Schwartz PJ, Napolitano C, et al: Risk stratification in the long-QT syndrome. N Engl J Med 2003;348:1866-1874.

15 Sansone V, Tawil R: Management and treatment of Andersen-Tawil syndrome (ATS). Neurotherapeutics 2007;4:233-237.

16 Tawil R, Ptacek LJ, Pavlakis SG, et al: Andersen's syndrome: potassium-sensitive periodic paralysis, ventricular ectopy, and dysmorphic features. Ann Neurol 1994;35:326-330

17 Bellocq C, van Ginneken AC, Bezzina CR, et al: Mutation in the KCNQ1 gene leading to the short QT-interval syndrome. Circulation 2004;109:2394-2397.

18 Priori SG, Pandit SV, Rivolta I, et al: A novel form of short QT syndrome (SQT3) is caused by a mutation in the KCNJ2 gene. Circ Res 2005;96:800-807.

19 Gollob MH, Redpath CJ, Roberts JD: The short QT syndrome: proposed diagnostic criteria. J Am Coll Cardiol 2011;57:802-812.

20 Goldenberg I, Horr S, Moss AJ, et al: Risk for life-threatening cardiac events in patients with genotype-confirmed long-QT syndrome and normal-range corrected QT intervals. J Am Coll Cardiol 2011;57:51-59.

21 Wong JA, Gula LJ, Klein GJ, Yee R, Skanes AC, Krahn AD: Utility of treadmill testing in identification and genotype prediction in long-QT syndrome. Circ Arrhythm Electrophysiol 2010;3:120-125.

22 Sy RW, van der WerfC, Chattha IS, et al: Derivation and validation of a simple exercisebased algorithm for prediction of genetic testing in relatives of LQTS probands. Circulation 2011;124:2187-2194.

23 Shimizu W, Noda T, Takaki H, et al: Diagnostic value of epinephrine test for genotyping LQT1, LQT2, and LQT3 forms of congenital long QT syndrome. Heart Rhythm 2004;1:276-283.

24 Ackerman MJ, Khositseth A, Tester DJ, Hejlik JB, Shen WK, Porter CB: Epinephrineinduced QT interval prolongation: a genespecific paradoxical response in congenital long QT syndrome. Mayo Clin Proc 2002;77: 413-421.

25 Shimizu W, Noda T, Takaki H, et al: Epinephrine unmasks latent mutation carriers with LQT1 form of congenital long-QT syndrome. J Am Coll Cardiol 2003;41:633-642.

26 Hekkala AM, Swan H, Viitasalo M, Vaananen H, Toivonen L: Epinephrine bolus test in detecting long QT syndrome mutation carriers with indeterminable electrocardiographic phenotype. Ann Noninvasive Electrocardiol 2011;16:172-179.

$\checkmark 27$ Kapa S, Tester DJ, Salisbury BA, et al: Genetic testing for long-QT syndrome: distinguishing pathogenic mutations from benign variants. Circulation 2009;120:1752-1760.

28 Ackerman MJ, Priori SG, Willems S, et al: HRS/EHRA expert consensus statement on the state of genetic testing for the channelopathies and cardiomyopathies: this document was developed as a partnership between the Heart Rhythm Society (HRS) and the European Heart Rhythm Association (EHRA). Europace 2011;13:1077-1109.

29 Gollob MH, Blier L, Brugada R, et al: Recommendations for the use of genetic testing in the clinical evaluation of inherited cardiac arrhythmias associated with sudden cardiac death: Canadian Cardiovascular Society/Canadian Heart Rhythm Society joint position paper. Can J Cardiol 2011;27:232245 
-30 Bhandari AK, Shapiro WA, Morady F, Shen EN, Mason J, Scheinman MM: Electrophysiologic testing in patients with the long QT syndrome. Circulation 1985;71:63-71.

-31 Giustetto C, Di Monte F, Wolpert C, et al: Short QT syndrome: clinical findings and diagnostic-therapeutic implications. Eur Heart J 2006;27:2440-2447.

32 Goldenberg I, Moss AJ: Long QT syndrome. J Am Coll Cardiol 2008;51:2291-2300.

33 Goldenberg I, Moss AJ, Peterson DR, et al: Risk factors for aborted cardiac arrest and sudden cardiac death in children with the congenital long-QT syndrome. Circulation 2008;117:2184-2191.

34 Goldenberg I, Moss AJ, Bradley J, et al: LongQT syndrome after age 40. Circulation 2008; 117:2192-2201.

- 35 Goldenberg I, Thottathil P, Lopes CM, et al: Trigger-specific ion-channel mechanisms, risk factors, and response to therapy in type 1 long QT syndrome. Heart Rhythm 2012;9: 49-56.

36 Goldenberg I, Mathew J, Moss AJ, et al: Corrected QT variability in serial electrocardiograms in long QT syndrome: the importance of the maximum corrected QT for risk stratification. J Am Coll Cardiol 2006;48:10471052.

37 Hobbs JB, Peterson DR, Moss AJ, et al: Risk of aborted cardiac arrest or sudden cardiac death during adolescence in the long-QT syndrome. JAMA 2006;296:1249-1254.

- 38 Jons C, Moss AJ, Goldenberg I, et al: Risk of fatal arrhythmic events in long QT syndrome patients after syncope. J Am Coll Cardiol 2010;55:783-788.

39 Zareba W, Moss AJ, Locati EH, et al: Modulating effects of age and gender on the clinical course of long QT syndrome by genotype. J Am Coll Cardiol 2003;42:103-109.

40 Locati EH, Zareba W, Moss AJ, et al: Ageand sex-related differences in clinical manifestations in patients with congenital longQT syndrome: findings from the International LQTS Registry. Circulation 1998;97: 2237-2244
41 Migdalovich D, Moss AJ, Lopes CM, et al: Mutation and gender-specific risk in type 2 long QT syndrome: implications for risk stratification for life-threatening cardiac events in patients with long QT syndrome. Heart Rhythm 2011;8:1537-1543.

42 Sauer AJ, Moss AJ, McNitt S, et al: Long QT syndrome in adults. J Am Coll Cardiol 2007; 49:329-337.

43 Gaita F, Giustetto C, Bianchi F, et al: Short QT syndrome: pharmacological treatment. J Am Coll Cardiol 2004;43:1494-1499.

44 Moss AJ, Zareba W, Hall WJ, et al: Effectiveness and limitations of beta-blocker therapy in congenital long-QT syndrome. Circulation 2000;101:616-623.

45 Vincent GM, Schwartz PJ, Denjoy I, et al: High efficacy of beta-blockers in long-QT syndrome type 1: contribution of noncompliance and QT-prolonging drugs to the occurrence of beta-blocker treatment 'failures'. Circulation 2009;119:215-221.

-46 Priori SG, Napolitano C, Schwartz PJ, et al: Association of long QT syndrome loci and cardiac events among patients treated with beta-blockers. JAMA 2004;292:1341-1344.

47 Zareba W, Moss AJ, Daubert JP, Hall WJ, Robinson JL, Andrews M: Implantable cardioverter defibrillator in high-risk long QT syndrome patients. J Cardiovasc Electrophysiol 2003;14:337-341.

48 Maron BJ, Chaitman BR, Ackerman MJ, et al: Recommendations for physical activity and recreational sports participation for young patients with genetic cardiovascular diseases. Circulation 2004;109:2807-2816.

49 Goldenberg I, Bradley J, Moss A, et al: Betablocker efficacy in high-risk patients with the congenital long-QT syndrome types 1 and 2: implications for patient management. J Cardiovasc Electrophysiol 2010;21:893901.

50 Fabritz L, Damke D, Emmerich M, et al: Autonomic modulation and antiarrhythmic therapy in a model of long QT syndrome type 3. Cardiovasc Res 2010;87:60-72.

51 Groh WJ, Silka MJ, Oliver RP, Halperin BD, McAnulty JH, Kron J: Use of implantable cardioverter-defibrillators in the congenital long QT syndrome. Am J Cardiol 1996;78: 703-706.
Epstein AE, Dimarco JP, Ellenbogen KA, et al: ACC/AHA/HRS 2008 guidelines for device-based therapy of cardiac rhythm abnormalities. Heart Rhythm 2008;5:e1-e62.

53 Monnig G, Kobe J, Loher A, et al: Implantable cardioverter-defibrillator therapy in patients with congenital long-QT syndrome: a long-term follow-up. Heart Rhythm 2005;2: 497-504.

54 Schwartz PJ, Priori SG, Cerrone M, et al: Left cardiac sympathetic denervation in the management of high-risk patients affected by the long-QT syndrome. Circulation 2004; 109:1826-1833.

55 Eldar M, Griffin JC, Van Hare GF, et al: Combined use of beta-adrenergic blocking agents and long-term cardiac pacing for $\mathrm{pa}$ tients with the long QT syndrome. J Am Coll Cardiol 1992;20:830-837.

56 Viskin S: Cardiac pacing in the long QT syndrome: review of available data and practical recommendations. J Cardiovasc Electrophysiol 2000;11:593-600.

57 Chaitman BR: Ranolazine for the treatment of chronic angina and potential use in other cardiovascular conditions. Circulation 2006;113:2462-2472.

58 Moss AJ, Zareba W, Schwarz KQ, Rosero S, McNitt S, Robinson JL: Ranolazine shortens repolarization in patients with sustained inward sodium current due to type-3 long-QT syndrome. J Cardiovasc Electrophysiol 2008; 19:1289-1293.

59 Moss AJ, Windle JR, Hall WJ, et al: Safety and efficacy of flecainide in subjects with long QT-3 syndrome (DeltaKPQ mutation): a randomized, double-blind, placebo-controlled clinical trial. Ann Noninvasive Electrocardiol 2005;10:59-66.

60 Khan IA, Nair CK: Brugada and long QT-3 syndromes: two phenotypes of the sodium channel disease. Ann Noninvasive Electrocardiol 2004;9:280-289.

61 Kobza R: Identifying risk of cardiac events in short QT syndrome. Heart Rhythm 2012;9: $75-76$. 